conducted with the aid of a grant from the British Empire Cancer Campaign.

Medical Research Council

Radiotherapeutic Research Unit, Hammersmith Hospital,

Radiotherapy Department, Hammersmith Hospital, Ducane Road,

Shepherds Bush,

London, W.12.

Oct. 10.

${ }^{1}$ Miller, D. R., Thompson, R. C., and Cunningham, B. B., Phys. Rex. 74, 347 (1948).

${ }^{2}$ Friedlander, G., and Miller, J. M., quoted by Strominger, D., Hol-

lander, M., and Seaborg, G. T., Rev. Mod. Phys, 30, 585 (1958)

${ }^{3}$ Arbman, E., and Svartholm, N., Arkiv Fysik, 10, 1 (1956).

+ Friedlander, G., and Miller, J. M., Phys. Rev., 84, 588 (1951).

${ }^{5}$ Osborne, R. K., and Deutsch, M., Phys. Rev., 71, 467A (1947).

\section{Use of Ion Exchange Resins in preparing Water of High Purity}

A RECENT communication ${ }^{1}$ directed the attention of research workers to the fact that de-ionized water could contain traces of colloidal polyelectrolytes derived from the anion exchange resin, and that the presence of such particles in the effuent could have an effect upon the properties of monolayers and impose a positive charge upon the walls of glass cells.

In the vast majority of applications the presence of minute traces of organic nitrogenous material is of no practical significance, and the remarkable ionic purity of mixed-bed de-ionized water, coupled with the ease of its preparation and its cheapness, makes it far more attractive than distilled water, which nearly always contains carbon dioxide and silica together with varying significant traces of metallic ions.

Where 'pure' water is required for work of the most critical nature, a mixed-bed ion exchange column may be used providing certain precautions are taken.

In the first place, fresh resins as obtained from manufacturers should not be used. In particular, the anion exchange resin should first be eluted and then alternately regenerated with caustic soda and exhausted with sodium chloride solution two or three times. In this way, degenerated nitrogenous materials remaining on the resin from the manufacturing process are removed. The cation exchange resins should be similarly treated by being passed through the hydrogen-sodium cycle.

After complete regeneration the resins should be thoroughly washed with distilled water (100 gallons/ cu. $\mathrm{ft}$. anion resin and 75 gallons/cu. $\mathrm{ft}$. cation resin) at a flow rate of 0.5 gallon $/ \mathrm{cu}$. ft. $/ \mathrm{min}$. and should then be intimately air-mixed in the proportion 2 anion resin : 1 cation resin, by volume.

The column should be of polyethylene, 'Perspex' or 'Pyrex' (not soda glass) and should be not less than 3 in. in diameter. A minimum of depth of bed of 24 in. should be used. In a narrow column the resinglass interface surface-volume ratio is poor and in a short column the contact time at the optimum flow rate is inadequate. The bottom filter should be of close-woven nylon or of a similar material, or else consist of a No. 1 porosity sintered 'Pyrex' disk.

The effluent should be passed through a further small column containing sulphonated polystyrene resin in the hydrogen form eross-linked 1-2 per cent.
Alternatively, the filter may take the form of a 'Pyrex' or polyethylene tube loosely packed with platinum wool maintained at a high negative potential above earth.

A particle of water introduced at the top of a column may reach the bottom in any of the following three ways : (1) by migration through the interstitial bead lattices; (2) by leakage between the beads; (3) by leakage between the resin column and the container walls.

Of these three methods of transit the first is clearly the only desirable one, and the probability of this method of transit occurring is favoured by using a column having a large volume-wall surface ratio and an adequate length.

In the lowest resin zones the column ceases to behave as a mono-bed owing to the random distribution of resin beads, with the result that binary and tertiary groups formed by the degeneration of quaternary ammonium groups may hydrolyse and go into solution. The cation resin filter will remove such components either by surface effect or by ion exchange, with the production of water. Colloidal particles of anionic resin will tend to be similarly adsorbed. Such particles will also be attracted to and retained upon a negatively charged surface.

The County Water Softener Co., Ltd.,

I. RALEIGiH

34 Victoria Street,

London, S.W.1.

Oct. 22.

schenkel, J. 11., and Kitchener, J. A., Nature, 182, 131 (195x)

THE instructions which Mr. Raleigh has given for preparing de-ionized water of high purity are valuable and timely; they should be of interest to research workers in many fields. Evidently, with a little extra trouble one can now consistently prepare water of markedly higher purity than is commonly obtained from small laboratory mixed-bed columns, and research workers requiring water of extremely low ionic content should obviously take this extra trouble. But caution is still needed in using such water for. critical studies.

First, the conductivity of the water should always be checked, using an apparatus suitable for resistivities around 5-20 megohm. cm. ${ }^{-1}$ (which manyconductivity meters are not), and its value should be stated in published work as proof of the quality of the water. The 'best' de-ionized water ${ }^{1.2}$ has a conductivity of about $0.07 \mu \mathrm{mho} \mathrm{cm}^{-1}$ at $20^{\circ} \mathrm{C}$., compared with $0 \cdot 1-0 \cdot 5$ commonly obtained from small mixed-bed columns. I suggest that 'high quality de-ionized water' be defined as having a specific conductivity of not more than $0 \cdot 1 \mu \mathrm{mho} \mathrm{cm} .^{-1}$

Secondly, our original warning ${ }^{3}$ against the use of de-ionized water for research in surface chemistry must still stand, even for high-quality water. The reason is that it still contains unknown, uncontrolled traces of surface-active substances of high molecular weight. In ordinary de-ionized water these impurities can be detected by chemical analysis and by their effects on surface tension and on the surface charge of glass. The amounts present in high-quality water are doubtless much smaller, but they constitute an insidious source of danger because they can accum. ulate at certain surfaces. A rough calculation of the mass of a monolayer sufficient to lower the surface tension of water in a Langmuir trough by 1 dyne $\mathrm{cm}^{-1}$ (an easily measurable effect) shows that it amounts to only about 0.006 part per million parts 